Article

\title{
Sustained Surface ICAM-1 Expression and Transient PDGF-B Production by Phorbol Myristate Acetate-Activated THP-1 Cells Harboring Blau Syndrome-Associated NOD2 Mutations
}

\author{
Mizuho Nishiyama ${ }^{1}$, Hong-jin Li ${ }^{1}$, Ikuo Okafuji ${ }^{2}$, Akihiko Fujisawa ${ }^{3}$, Mizue Ehara ${ }^{4}$, Naotomo Kambe ${ }^{3,4}$, \\ Fukumi Furukawa ${ }^{1}$ and Nobuo Kanazawa ${ }^{1,5, * \text { (D) }}$
}

1 Department of Dermatology, School of Medicine, Wakayama Medical University, Wakayama 641-0012, Japan; mizmiz254-med@yahoo.co.jp (M.N.); lhj215@163.com (H.-j.L.); dajs@wakayama-med.ac.jp (F.F.)

2 Department of Pediatrics, Kobe City Medical Center General Hospital, Kobe 650-0047, Japan; ikuoka@gmail.com

3 Department of Dermatology, Graduate School of Medicine, Kyoto University, Kyoto 606-8507, Japan; akihiko@kuhp.kyoto-u.ac.jp (A.F.); nkambe@kuhp.kyoto-u.ac.jp (N.K.)

4 Department of Dermatology, Graduate School of Medicine, Chiba University, Chiba 260-8670, Japan; ehara@faculty.chiba-u.jp

5 Department of Dermatology, School of Medicine, Hyogo College of Medicine, Nishinomiya 663-8501, Japan

* Correspondence: nkanazaw@hyo-med.ac.jp

Citation: Nishiyama, M.; Li, H.-j.; Okafuji, I.; Fujisawa, A.; Ehara, M.; Kambe, N.; Furukawa, F.; Kanazawa, N. Sustained Surface ICAM-1 Expression and Transient PDGF-B Production by Phorbol Myristate Acetate-Activated THP-1 Cells Harboring Blau SyndromeAssociated NOD2 Mutations. Children 2021, 8, 335. https://doi.org/ 10.3390/children8050335

Academic Editor: Maurizio Elia

Received: 31 December 2020

Accepted: 21 April 2021

Published: 25 April 2021

Publisher's Note: MDPI stays neutral with regard to jurisdictional claims in published maps and institutional affiliations.

Copyright: (c) 2021 by the authors. Licensee MDPI, Basel, Switzerland. This article is an open access article distributed under the terms and conditions of the Creative Commons Attribution (CC BY) license (https:// creativecommons.org/licenses/by/ $4.0 /)$.

\begin{abstract}
Objectives: Blau syndrome is a distinct class of autoinflammatory syndrome presenting with early-onset systemic granulomatosis. Blau syndrome-causing NOD2 mutations located in the central nucleotide-oligomerization domain induce ligand-independent basal NF- $\mathrm{kB}$ activation in an in vitro reporter assay. However, the precise role of this signaling on granuloma formation has not yet been clarified. Methods: Blau syndrome-causing NOD2 mutations were introduced into human monocytic THP-1 cells, and their morphological and molecular changes from parental cells were analyzed. Identified molecules with altered expression were examined in the patient's lesional skin by immunostaining. Results: Although the production of proinflammatory cytokines was not altered without stimulation, mutant NOD2-expressing THP-1 cells attached persistently to the culture plate after stimulation with phorbol myristate acetate. Sustained surface ICAM-1 expression was observed in association with this phenomenon, but neither persistent ICAM-1 mRNA expression nor impaired ADAM17 mRNA expression was revealed. However, the transient induction of PDGF-B mRNA expression was specifically observed in stimulated THP-1 derivatives. In the granulomatous skin lesion of a Blau syndrome patient, ICAM-1 and PDGF-B were positively immunostained in NOD2-expressing giant cells. Conclusions: Sustained surface ICAM-1 expression and transient PDGF-B production by newly differentiating macrophages harboring mutant NOD2 might play a role in granuloma formation in Blau syndrome.
\end{abstract}

Keywords: Blau syndrome; NOD2 mutation; ICAM-1; PDGF-B

\section{Introduction}

Autoinflammatory diseases constitute a group of genetic disorders whose main clinical features are recurrent episodes of inflammatory lesions that can affect the skin, joints, bones, eyes, gastrointestinal tract and nervous system, in association with signs of systemic inflammation $[1,2]$. Blau syndrome is a distinct class of autoinflammatory syndrome showing early-onset systemic granulomatosis [3-5]. In comparison to sarcoidosis, which is a multi-organ granulomatous disease with unknown etiology, Blau syndrome is histologically undistinguishable but clinically distinguished by the triad of skin rash, uveitis and arthritis without apparent lung involvement.

Blau syndrome has been shown to be caused by heterozygous missense mutations in the NOD2/CARD15/NLRC2 gene $[3,6]$. NOD2 is a member of the NOD-like receptor 
(NLR) family of molecules and is composed of two amino-terminal caspase recruitment domains (CARDs), a centrally located nucleotide-binding oligomerization domain (NOD) and carboxy-terminal leucine-rich repeats (LRR). This molecule is expressed intracellularly in antigen-presenting cells (APC) and recognizes muramyl dipeptide (MDP), the minimum common component of bacterial cell wall peptidoglycan, to form a complex with CARDcontaining serine/threonine kinase, RICK, and to induce immune responses through nuclear factor (NF)- $\mathrm{kB}$ activation $[4,7]$. NF- $\mathrm{kB}$ is one of the most important transcription factors inducing the expression of various cytokines, growth factors and cell adhesion molecules, and plays critical roles in developmental/survival and inflammatory processes [8]. Thus, NOD2 functions as an intracellular sensor for bacterial invasion. While mutations in the LRR impairing MDP-dependent NF- $\mathrm{KB}$ activation are reportedly associated with Crohn's disease (CD), Blau syndrome-associated NOD2 variants are localized in the NOD and show increased MDP-independent basal NF- $\mathrm{KB}$ activation, which is measured by an in vitro reporter assay using HEK293 cells [3,9-11]. By analysis of nine types of NOD2 mutations collected from 20 Japanese Blau syndrome patients, the basal NF$\kappa \mathrm{B}$ activity level, which was presented as a ratio of the NF- $\mathrm{KB}$ activity in the absence of MDP to that in the presence of MDP, was shown to range from 0.3 for the E383G mutation to 0.9 for the N670K mutation, compared to 0.05 for the wild-type [12]. Although a clear correlation was reportedly absent between clinical severity and the basal NF- $\mathrm{kB}$ activation level of the corresponding NOD2 mutation, a tendency was observed when the subjects were limited to ocular complications in cases with frequent R334W and R334Q mutations [12]. Furthermore, the therapeutic effect for Blau syndrome of thalidomide, a potent immunomodulatory drug suppressing NF- $\mathrm{KB}$ activation, may also suggest the role of NF- $\mathrm{KB}$ in the pathogenesis of this disease [13].

Granulomatous reaction is a distinct pathological pattern of chronic inflammation forming granuloma, and is classified into several types: foreign body, suppurative, tuberculoid, palisaded, interstitial and sarcoidal [14]. Sarcoidal granuloma is considered to be caused by a super-delayed hypersensitivity reaction directed at any unknown antigen. The release of inflammatory mediators, especially interferon (IFN)- $\gamma$, from activated T helper (Th) 1 cells is considered to be indispensable for the activation and accumulation of macrophages and subsequent granuloma formation [15]. In the case of pulmonary sarcoidosis, underlying latent infection with some distinct microorganism, such as Propionibacterium acnes and Mycobacterium species, has been shown [16]. In addition, some foreign substance such as silica can be found in the case of scar sarcoidosis [17]. These facts suggest the role of infection and/or innate immunity in the development of sarcoidal granuloma.

As suggested by the results of in vitro experimental findings, constitutive NF- $\mathrm{kB}$ activation in NOD2-expressing APC is considered responsible for granuloma formation in Blau syndrome. However, the precise role of such NF- $\mathrm{kB}$ activation and subsequent changes are still undefined. Therefore, to better understand these issues, NOD2 mutations caused by Blau syndrome were introduced into human monocytic THP- 1 cells to generate another in vitro model resembling monocytes. Any alterations from parental cells were identified by morphological and molecular analyses. Furthermore, the expression of identified intercellular cell adhesion molecule (ICAM)-1 with a sustained surface expression and platelet-derived growth factor (PDGF)-B with a transiently-induced production by phorbol myristate acetate (PMA)-treated THP-1 derivatives was analyzed in the lesional skin of a Blau syndrome patient by immunostaining.

\section{Materials and Methods}

\subsection{Cell Culture}

THP-1 cells were originally purchased from ATCC (American Type Culture Collection, Manassas, VA, USA) and maintained in RPMI1640 supplemented with 10\% fetal bovine serum as previously described [18]. Transfected THP-1 derivatives were maintained in the presence of $500 \mu \mathrm{g} / \mathrm{mL}$ G418 (Invitrogen, Carlbad, CA, USA) to avoid the excessive proliferation of wild-type cells. 


\subsection{Reagents and Antibodies}

PMA was purchased from Sigma-Aldrich (St. Louis, MO, USA). Human recombinant interleukin (IL)-4 and macrophage-colony stimulation factor (M-CSF) were purchased from Peprotech (Rocky Hill, NJ, USA). Mouse anti-FLAG M2 (Sigma-Aldrich), ICAM-1 (Immunotech), tumor necrosis factor (TNF) $\alpha$ (Santa Cruz Biotechnology, Inc. Dallas, TX, USA), NOD2 and interferon (IFN) $\gamma$ (eBioscience) monoclonal antibodies (mAbs) were purchased. Rabbit anti-cadherin-11 (Zymed), NOD2 (Sigma-Aldrich) and PDGF-B (Novus Biologicals) polyclonal Abs were also purchased.

\subsection{Transfection}

The complementary DNAs (cDNAs) for wild-type (WT), R334W and N670K mutant $N O D 2$ were digested from the corresponding plasmids in the p3xFLAG-CMV vector, which were previously used for the NF- $\mathrm{kB}$ reporter assay, and recloned into the pIRES2-EGFP vector (Clontech) [3]. Each of these plasmids and the mock vector were linearized with AflII (New England Biolabs, Ipswich, MA, USA) and introduced into THP-1 cells using Amaxa Nucleofactor (Lonza Cologne GmbH, Cologne, Germany) according to the manufacturer's recommended protocol. The transfected cells were selected in the presence of $750 \mu \mathrm{g} / \mathrm{mL}$ of G418.

\subsection{RT-PCR Analysis}

Total mRNA was extracted from THP-1 derivatives using Sepasol (Nakarai Tesque, Kyoto, Japan), and $1 \mu \mathrm{g}$ of the mRNA was applied for cDNA synthesis using the Superscript first-strand synthesis kit including reverse transcriptase II and poly-T primer (Invitrogen). PCR was performed using Ex-Taq (Takara Bio Inc., Otsu, Japan) and ABI2720 thermal cycler (Thermo Fisher Scientific, Waltham, MA, USA) under the following conditions: $95^{\circ} \mathrm{C}$ for $5 \mathrm{~min}$ followed by 35 cycles of $94^{\circ} \mathrm{C}$ for $30 \mathrm{~s}, 57^{\circ} \mathrm{C}$ for $30 \mathrm{~s}$ and $72{ }^{\circ} \mathrm{C}$ for $1 \mathrm{~min}$, and finally $72{ }^{\circ} \mathrm{C}$ for $10 \mathrm{~min}$. Specific primer pairs are listed in Table 1.

Table 1. Primer pairs used for RT-PCR analyses.

\begin{tabular}{|l|l|l|}
\hline & Forward & Reverse \\
\hline NOD2 & AGACTCAGCTTCCCAAGGTCTG & AGAACACGTAGCAGCACATGCC \\
\hline $\mathbf{I L - 8}$ & AAGGAATAGCATCAATAGTGAGTTTG & GGACACAAGCTTAAACCCAGA \\
\hline ICAM-1 & CCTTCCTCACCGTGTACTGG & AGCGTAGGGTAAGGTTCTTGC \\
\hline ADAM17 & CCTTTCTGCGAGAGGGAAC & CACCTTGCAGGAGTTGTCAG \\
\hline PDGF-B & CCTTTGATGATCTCCAACGC & GATCTTTCTCACCTGGACAG \\
\hline HPRT & AATTATGGACAGGACTGAACGTC & CGTGGGGTCCTTTTCACCAGCAAG \\
\hline
\end{tabular}

\subsection{Flow Cytometry}

THP-1 derivatives were stained with primary antibody or the corresponding isotype control and the subsequent appropriate secondary antibodies, and were provided for analysis with FACSCaliber and BD CellQuest ${ }^{\mathrm{TM}}$ Pro software (BD Biosciences, San Jose, CA, USA).

\subsection{Immunohistochemistry}

10- $\mu$ m-thick sections of formaldehyde-fixed paraffin-embedded skin biopsy specimens, which were obtained from a Blau syndrome patient with a R334W mutation, were deparaffinized and subjected to staining with mouse monoclonal anti-ICAM-1, TNF $\alpha$, IFN $\gamma$ or rabbit polyclonal anti-PDGF-B Abs or the control mouse IgG1 or rabbit immunoglobulin. Positive staining was visualized using the VECTASTAIN Elite ABC Kit (Vector Laboratories, Inc., Burlingame, CA, USA) and DAB substrate kit (Dako Denmark A/S, Glostrup, 
Denmark) according to the manufacturer's protocol. The sections were counterstained with hematoxylin.

\subsection{Double Immunofluorescence Staining}

The Blau syndrome patient's skin specimen was applied for double immunofluorescence staining with a set of rabbit polyclonal anti-NOD2 Abs and mouse monoclonal anti-ICAM-1 Abs or a set of mouse monoclonal anti-NOD2 Abs and rabbit polyclonal anti-PDGF-B Abs at 1:50 dilution. The specimen was then stained with Alexa Fluor 488conjugated anti-rabbit IgG, Alexa Fluor 555-conjugated anti-mouse IgG and Hoechst 33342. Sections were mounted with Vectashield (Vector Laboratories, Inc.) and observed with confocal microscopy (LSM780, Carl Zeiss Microscopy, Jena, Germany).

\section{Results}

3.1. No Altered mRNA Expression of Proinflammatory Cytokines in Mutant NOD2-Expressing THP-1 Derivatives

THP-1 cells were transfected by electroporation with either mock, FLAG-tagged WT, R334W or N670K mutant NOD2 cloned in the pIRES2-EGFP vector. Among colonies developing in the presence of G418 at the manufacturer's recommended concentration (750 $\mu \mathrm{g} / \mathrm{mL}$ ), those expressing EGFP and intracellular FLAG were selected by flow cytometry and were subsequently analyzed with RT-PCR for NOD2 mRNA expression. Although NOD2 mRNA expression was only faintly observed in mock-transfected THP-1 cells, significant expression was observed in WT or mutant NOD2-transfected THP-1 derivatives. For each derivative, one representative colony showing an almost equal expression of NOD2 mRNA was selected and used for further analyses (Figure 1). The predominant expression of the mutant NOD2 in the corresponding colony was confirmed by direct sequencing of the RT-PCR product (data not shown). Without stimulation, the expression of mRNA for proinflammatory cytokines such as TNF $\alpha$ and IL- 8 was similar for WT and mutant NOD2-expressing THP-1 derivatives (Figure 1).

\subsection{Long-Term Attachment of Mutant NOD2-Expressing THP-1 Derivatives after PMA Stimulation}

Following PMA stimulation, THP-1 cells are known to differentiate along the monocytic lineage and to acquire characteristics of mature macrophages, including a loss of proliferation and an increased HLA-DR expression [19,20]. A large difference was morphologically observed between WT and mutant NOD2-expressing THP-1 derivatives after PMA stimulation. As shown in Figure 2, all THP-1 derivatives were attached to the culture plate and spread pseudopods $24 \mathrm{~h}$ after PMA addition. However, on day 3, mock or WT NOD2-expressing THP-1 derivatives floated into the medium again and proliferated, whereas mutant NOD2-expressing THP-1 derivatives remained attached to the plate and spread more and longer pseudopods. Such characteristic features were still apparent on day 7, especially in the case of N670K mutant NOD2-expressing THP-1 derivatives, as made clear after washing (bottom column of Figure 2). In contrast, R334W mutant NOD2-expressing THP-1 derivatives floated into the medium again and proliferated on day 7. 


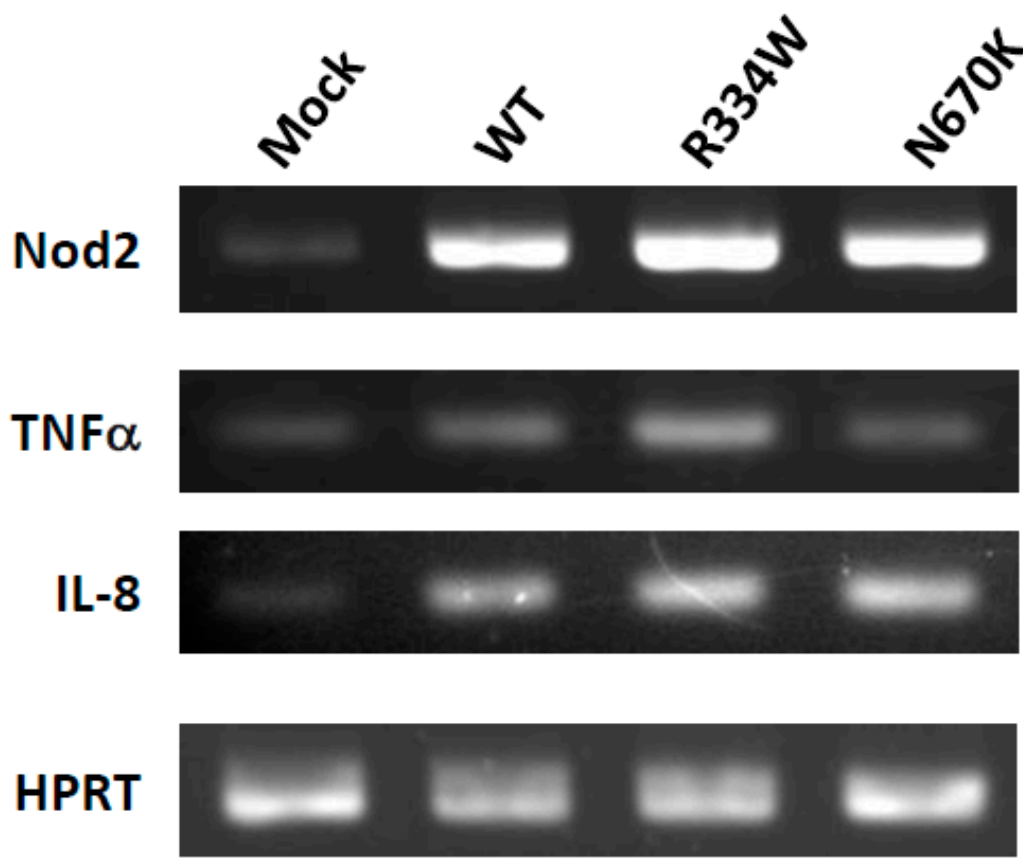

Figure 1. No altered mRNA expression of proinflammatory cytokines in mutant NOD2-expressing THP-1 derivatives. THP-1 cells were transfected with mock, WT, R334W or N670K mutant NOD2 cDNA-containing letroviral vector and were selected for study in the presence of G418. From the surviving colonies, THP-1 derivatives with similar expression levels of NOD2 mRNA revealed by RT-PCR were selected for further analyses (top row). Expression of TNF $\alpha$ or IL- 8 mRNA in these derivatives was then analyzed by RT-PCR without stimulation. Hypoxanthine phosphoribosyltransferase (HPRT) was analyzed as a control for ubiquitous expression (bottom row).

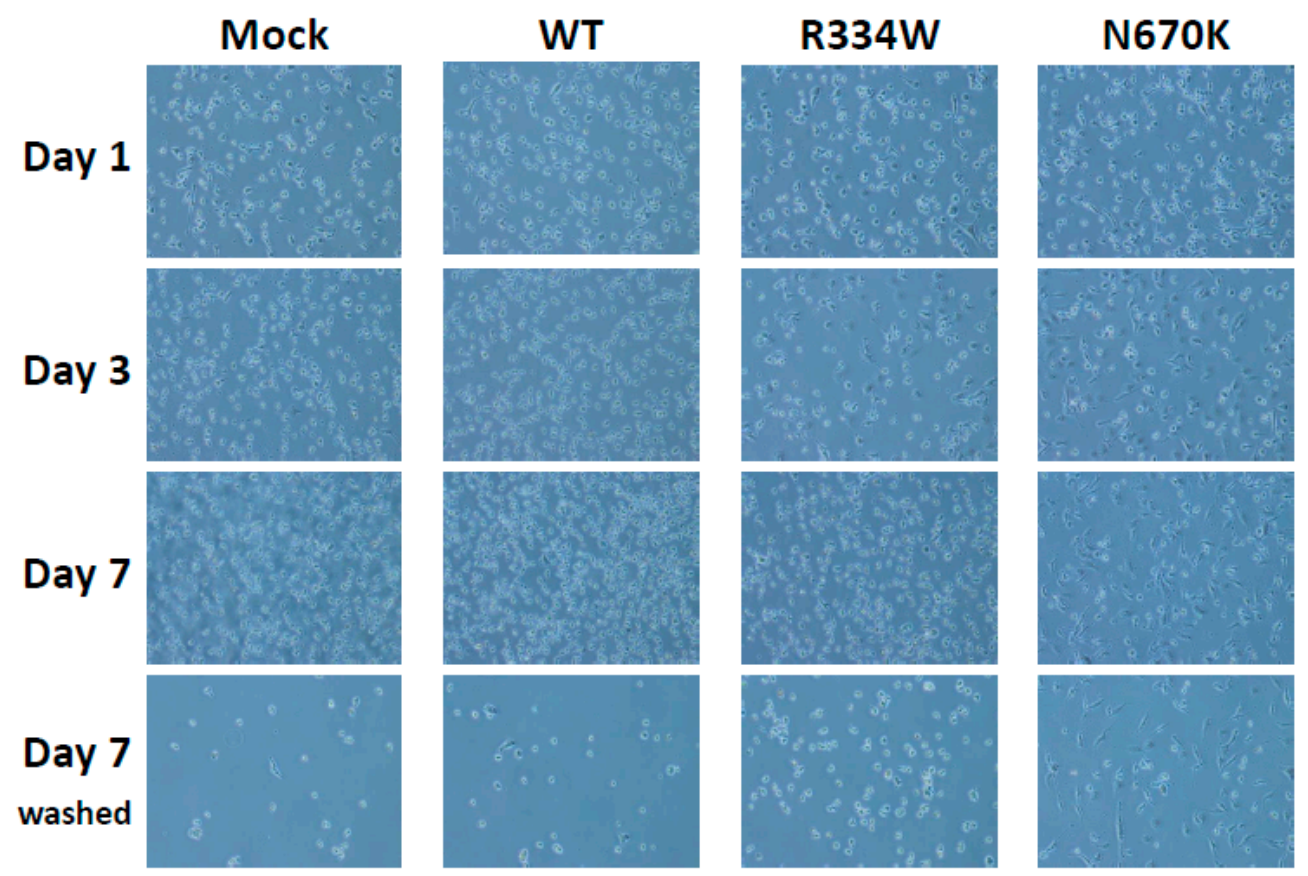

Figure 2. Long-term attachment of mutant NOD2-expressing THP-1 derivatives after PMA stimulation. THP-1 derivatives were stimulated with $10 \mathrm{mM}$ of PMA, and photographs were taken under bright field lightning at the indicated time points. On day 7 after PMA addition, photographs were again taken after washing the floating cells (bottom row). 


\subsection{Sustained Surface Expression of ICAM-1 on Mutant NOD2-Expressing THP-1 Derivatives}

To explore the mechanism underlying the long-term attachment of mutant NOD2expressing THP-1 derivatives after PMA stimulation, the surface expression levels of various adhesion molecules were analyzed by flow cytometry. As shown in Figure 3, the surface expression of ICAM-1 was initially upregulated in all THP-1 derivatives on day 2 and then decreased to almost the basal level in mock or WT NOD2-expressing THP-1 derivatives on day 6. However, the expression remained higher for mutant NOD2expressing THP-1 derivatives at this time point, even when considering the significant fluorescence alteration of the isotype control.

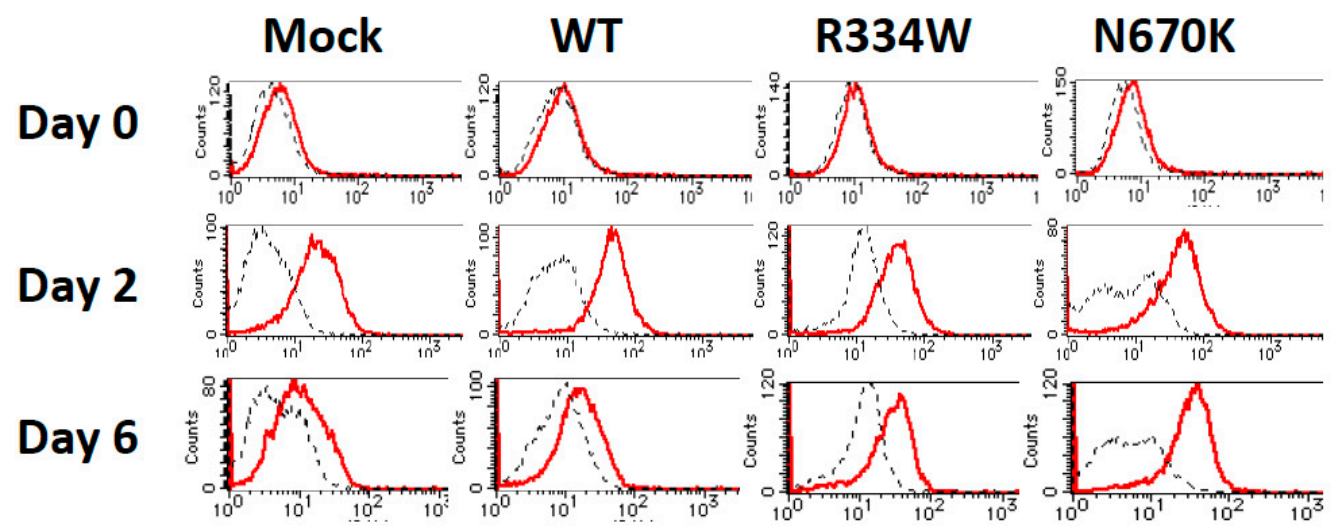

Figure 3. Sustained surface expression of ICAM-1 on mutant NOD2-expressing THP-1 derivatives. The surface expression of ICAM-1 on THP-1 derivatives was analyzed with flow cytometry at the indicated time points after PMA addition. The isotype control is shown by a dotted line.

3.4. No Remarkable Alteration of ICAM-1 or ADAM-17 mRNA Expression Underlies Sustained Surface Expression of ICAM-1

By RT-PCR analysis, ICAM-1 mRNA expression in all THP-1 derivatives was initially upregulated on day 2 and similarly decreased on day 6 after PMA addition, suggesting that the sustained surface expression of ICAM- 1 was not due to prolonged mRNA expression (Figure 4a, left column). We then analyzed the mRNA expression of ADAM-17, which reportedly mediates the cleavage of the ectodomain of ICAM-1 [21]. However, ADAM17 mRNA was similarly expressed in all unstimulated THP-1 derivatives, and no further increase was observed in any of the derivatives after PMA addition, suggesting that a defective ICAM-1 cleavage by ADAM-17 mRNA induction was not involved in sustained surface ICAM-1 expression (Figure 4a, middle column). 

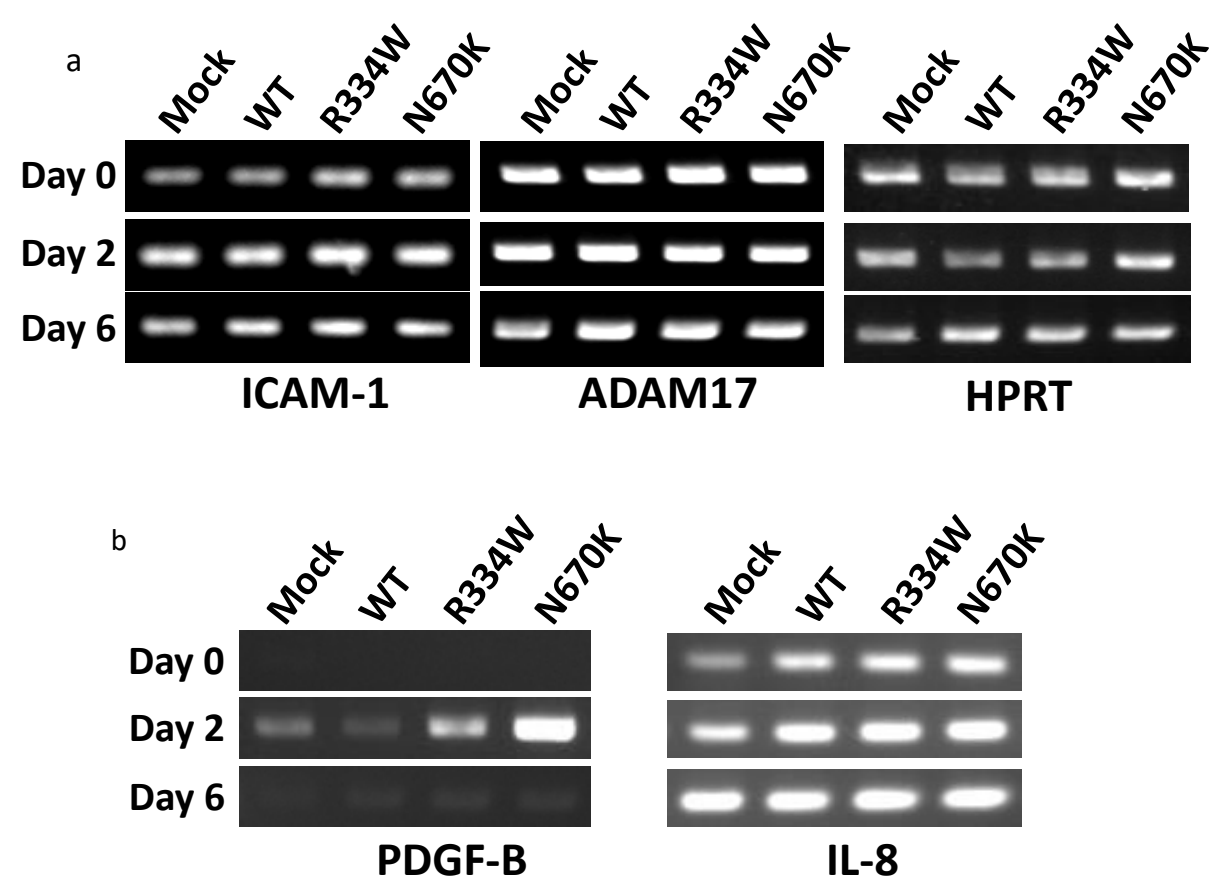

Figure 4. No altered ICAM-1 or ADAM-17 mRNA expression but a transient PDGF-B mRNA expression in PMA-stimulated mutant NOD2-expressing THP-1 derivatives. The expression of (a) ICAM-1 or ADAM-17 mRNA and (b) PDGF-B or IL-8 mRNA on THP-1 derivatives was analyzed by RT-PCR using the cells shown in Figure 3 and was compared between WT and mutant NOD2expressing derivatives at each time point. No remarkable difference was observed in the expression of ICAM-1, ADAM17 or IL-8.

\subsection{Transient PDGF-B mRNA Expression in PMA-Stimulated Mutant NOD2-Expressing THP-1 Derivatives}

To explore the mRNA specifically induced in PMA-stimulated NOD2-expressing THP1 cells, RT-PCR was performed for various cytokines and growth factors. Among them, mRNA expression of PDGF-B, which was undetectable in all unstimulated THP-1 derivatives, was strongly induced in mutant NOD2-expressing THP-1 derivatives, especially in the case of the N670K mutant, but only weakly induced in mock or WT NOD2-expressing cells on day 2 after PMA addition (Figure $4 \mathrm{~b}$, left column). PDGF-B is an essential growth factor involved in wound healing and might play a pivotal role in granuloma formation through activation of the surrounding fibroblasts. Notably, the induced PDGF-B mRNA expression was not sustained and decreased to the basal level in all THP-1 derivatives on day 6 after PMA addition (Figure 4b, left column). In contrast, IL-8 mRNA expression was induced to a similar extent on day 2 and was sustained on day 6 after PMA addition in all THP-1 derivatives, as shown in the right column of Figure $4 \mathrm{~b}$.

3.6. ICAM-1 and PDGF-B Protein Expression in NOD2-Expressing Giant Cells in the Lesional Skin of a Blau Syndrome Patient

To explore the site of ICAM-1 and PDGF-B expression and its relationship with NOD2 expression in Blau syndrome lesions, ICAM-1 and PDGF-B protein expression in the lesional skin specimen of a Blau syndrome patient harboring the NOD2 R334W mutation was examined immunohistochemically and by double-immunofluorescence staining with NOD2. By immunohistochemistry, ICAM-1 expression was observed linearly just beneath the surface of multinucleated giant cells (MGCs), while PDGF-B expression was observed diffusely within (cytoplamic or perinuclear) granuloma-forming epithelioid cells and MGCs, as shown in Figure 5a. In contrast, no expression of TNF $\alpha$ or IFN $\gamma$ was detected in the lesional skin (Figure 5a). By double-immunofluorescence staining, ICAM-1 and PDGF-B were weakly stained in MGCs and were both well co-localized with NOD2, which was clearly stained in the perinuclear region of MGCs (Figure 5b,c). 


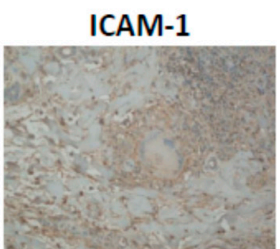

TNF $\alpha$

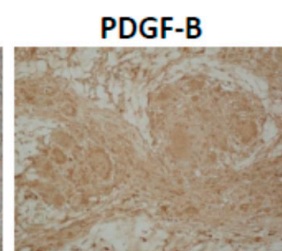

IFN $\gamma$

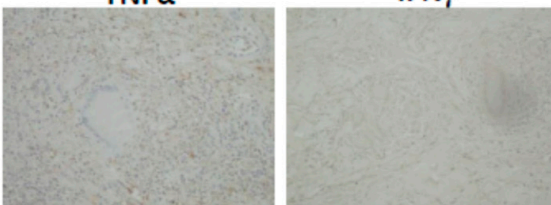

b
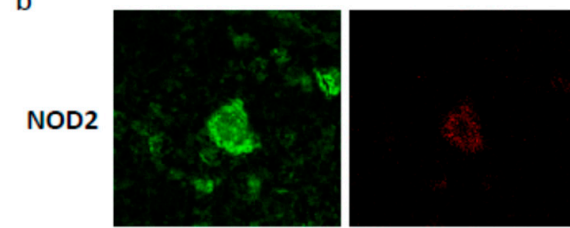

ICAM-1
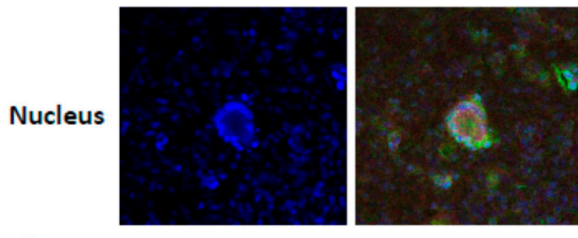

C
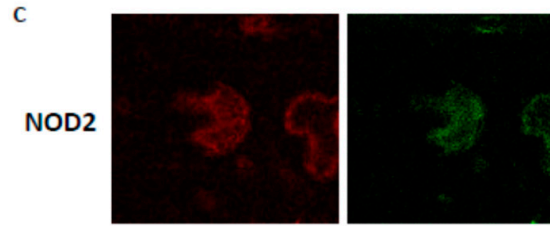

PDGF-B
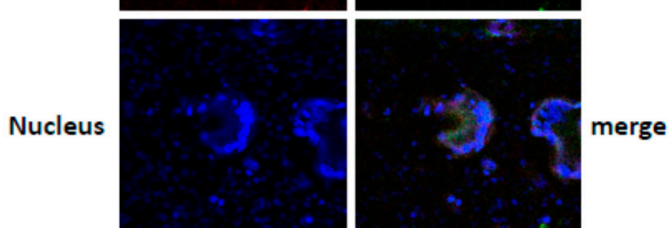

Figure 5. (a) Expression of ICAM-1, PDGF-B, TNF $\alpha$ or IFN $\gamma$ in the lesional skin of a Blau syndrome patient with a NOD2 R334W mutation was analyzed by immunohistochemistry. (Original magnification: $\times 100)$. $(\mathbf{b}, \mathbf{c})$ ICAM-1 and PDGF-B protein expression in NOD2-expressing giant cells in the lesional skin of a Blau syndrome patient. Expression of NOD2 (green) with ICAM-1 (red) or of NOD2 (red) with PDGF-B (green) was analyzed by double immunofluorescence and observed by confocal microscopy. Nuclei were stained by Hoechst 33342 and shown in blue. (Original magnification: $\times 200)$.

\section{Discussion}

Blau syndrome is a rare but genetically and histologically distinct disease, characterized by a gain-of-function NOD2 mutation and noncaseating epithelioid cell granuloma. Since monocytic cells with a NOD2 mutation are considered to play an essential role in granuloma formation in Blau syndrome, THP-1 cells of a human monocytic lineage were selected for a cellular model to analyze the pathogenesis of this disease. Therefore, it was unexpected that THP-1 cells expressing R334W or N670K-mutant NOD2 showed no upregulated production of TNF $\alpha$ or IL-8 (Figure 1). However, these results are consistent with a previous report showing normal or less responsiveness of Blau syndrome patients' peripheral blood mononuclear cells to various PAMP molecules [22]. Subsequently, we tried PMA, a strong NF- $\kappa B$ activator, to activate THP-1 cells. PMA is also known to induce the differentiation of THP- 1 cells into macrophage-like cells $[19,20]$. Indeed, all THP-1 derivatives changed their shape in a similar way immediately after PMA addition and differentiated into macrophage-like attaching cells. However, very surprisingly, mutant NOD2-expressing THP-1 derivatives further extended their pseudopods and remained attached to the plate at subsequent observations, whereas control THP- 1 cells began to float in the medium again and started to proliferate (Figure 2). These results suggest that the activating stimulus created by PMA is transient and induces regulatory pathways in the later stage. Since the surface expression of ICAM-1 on THP-1 derivatives seems to be correlated with their differentiation stage into macrophage-like cells (as shown in Figure 3), the downregulation of the surface ICAM-1 expression might be associated with the PMA-induced regulatory pathway. Further, this PMA-induced regulatory pathway might be abrogated by the Blau syndrome-causing NOD2 mutation. Although the alteration of mRNA expression due to NF- $\mathrm{kB}$ activation was expected, neither the mRNA of 
ICAM-1 itself or of its shedding enzyme ADAM-17 was significantly changed, as shown in Figure 4a. Notably, the putative correlation of the surface ICAM-1 expression and its mRNA expression remains to be elucidated more quantitatively. As the level of soluble ICAM-1 in bronchoalveolar lavage is reportedly correlated with the severity of sarcoidosis, ICAM-1 expression in monocytic cells seems to have some association with granuloma formation [23].

Another interesting point is the induction of PDGF-B, but not IL-8, mRNA in mutant NOD2-expressing THP-1 cells after PMA activation (Figure $4 \mathrm{~b}$ ). Although an exhaustive study would be required to clarify the specificity of PDGF-B induction, the specific expression of PDGF-B was also observed immunohistochemically in the lesional skin of a Blau syndrome patient, as shown in Figure 5a. Since NF- $\mathrm{kB}$ is reportedly capable of inducing both PDGF-B and IL-8 mRNA expressions, some regulatory mechanism or other specific transcription factor might be involved [24,25]. PDGF-B is a strong activator of fibroblasts and plays an important role in tissue repair. Interestingly, PDGF-B expression was detected as early as day 1 and persisted for at least 14 days in the cutaneous tuberculin reaction as an in vivo human model of T cell-mediated delayed hypersensitivity [26]. As is the case with sarcoidosis, which can finally cause lung fibrosis, overexpression of PDGF-B in mouse lungs reportedly induced a complex phenotype that encompassed both features of emphysema and fibrosis [27]. Although the role of PDGF-B in granuloma formation is unclear, it might work on preparing the fibrous network surrounding granulomas.

To generate MGCs, various PAMP molecules or uric acid crystals were added to the PMA-stimulated THP-1 derivatives. However, no fusion of the attached cells was observed (data not shown). Although it was reported that exposure of Blau syndrome patients' peripheral blood monocytes to IL-4 plus M-CSF caused their morphologic change into fibroblastic/dendritic cells with Langhans-type MGCs, neither fibroblastic/dendritic change nor MGC formation was induced in our THP-1 variants by this method [13].

Diffuse ICAM-1 expression along the surface of MGC and PDGF-B expression within granuloma-forming epithelioid cells and MGC formation in a Blau syndrome patient's skin specimen seem compatible with their predicted functions (Figure 5a). In contrast, no expression of TNF $\alpha$ or IFN $\gamma$ in the lesional skin was unexpected. Although the expression of both TNF $\alpha$ and IFN $\gamma$ in the lesional skin of a Blau syndrome patient was previously reported, the genetic background of the patient was not clarified [28]. This suggests a distinct mechanism for granuloma formation in Blau syndrome, possibly independent of $\mathrm{T}$ cells. To examine this possibility, the mutant NOD2-expressing THP-1 variants were injected subcutaneously into athymic (nu/nu) mice with or without PMA. However, they never formed epithelioid cell granulomas with MGCs in vivo (data not shown). The colocalization of ICAM-1 and PDGF-B with NOD2 seems consistent with our experimental results (Figure $5 b, c)$. However, it remains to be elucidated whether the expressed NOD2 mRNA in MGCs is mutated because the patient is heterozygous to the mutation.

Collectively, these observations suggest that sustained surface ICAM-1 expression on and transient PDGF-B production in newly differentiating macrophages that harbor a mutant NOD2 and respond to some stimuli might play a role in granuloma formation in Blau syndrome patients.

Author Contributions: Conceptualization, N.K. (Nobuo Kanazawa); methodology, A.F., N.K. (Naotomo Kambe) and N.K. (Nobuo Kanazawa); software, N.K. (Naotomo Kambe) and N.K. (Nobuo Kanazawa); validation, N.K. (Nobuo Kanazawa); formal analysis, N.K. (Nobuo Kanazawa); investigation, M.N., H.-j.L., A.F., M.E., N.K. (Naotomo Kambe) and N.K. (Nobuo Kanazawa); resources, N.K. (Naotomo Kambe) and N.K. (Nobuo Kanazawa); data curation, M.N. and N.K. (Nobuo Kanazawa); writing-original draft preparation, M.N.; writing-review and editing, N.K. (Nobuo Kanazawa); visualization, M.N. and N.K. (Nobuo Kanazawa); supervision, I.O., N.K. (Naotomo Kambe) and F.F.; project administration, N.K. (Nobuo Kanazawa); funding acquisition, N.K. (Naotomo Kambe) and N.K. (Nobuo Kanazawa). All authors have read and agreed to the published version of the manuscript. 
Funding: This research was funded in part by a grant from the Japan Society for Promotion of Science 19K08798 (N. Kanazawa) and Japan Ministry of Health, Labor and Welfare (N.K. (Naotomo Kambe) and N.K. (Nobuo Kanazawa)).

Institutional Review Board Statement: The study was conducted according to the guidelines of the Declaration of Helsinki and approved by the Ethics Committee of Kyoto University (G-68).

Informed Consent Statement: Informed consent was obtained from all subjects involved in the study.

Data Availability Statement: The data presented in this study are available on request from the corresponding author. The data are not publicly available due to patient's privacy.

Acknowledgments: We would like to thank Yumi Nakatani for technical assistance. This work was supported in part by a grant from the Japan Society for Promotion of Science (NK) and Japan Ministry of Health, Labor and Welfare.

Conflicts of Interest: The authors declare no conflict of interest.

\section{References}

1. Kanazawa, N.; Furukawa, F. Autoinflammatory syndromes with a dermatological perspective. J. Derm. 2007, 34, 601-618. [CrossRef] [PubMed]

2. Masters, S.L.; Simon, A.; Aksentijevich, I.; Kastner, D.L. Horror autoinflammaticus: The molecular pathophysiology of autoinflamatory disease. Annu. Rev. Immunol. 2009, 27, 621-668. [CrossRef] [PubMed]

3. Kanazawa, N.; Okafuji, I.; Kambe, N.; Nishikomori, R.; Nakata-Hizume, M.; Nagai, S.; Fugi, A.; Yuasa, T.; Manki, A.; Sakurai, Y.; et al. Early-onset sarcoidosis and CARD15 mutations with constitutive nuclear factor- $\mathrm{kB}$ activation: Common genetic etiology with Blau syndrome. Blood 2005, 205, 1195-1197.

4. Kambe, N.; Nishikomori, R.; Kanazawa, N. The cytosolic pattern-recognition receptor Nod2 and inflammatory granulomatous disease. J. Dermal. Sci. 2005, 39, 71-80. [CrossRef] [PubMed]

5. Rose, C.D.; Martin, T.M.; Wouters, C.H. Blau syndrome revisited. Curr. Opin. Rheumatol. 2011, 23, 411-418. [CrossRef]

6. Miceli-Richard, C.; Lesage, S.; Rybojad, M.; Prieur, A.M.; Manouvrier-Hanu, S.; Hafner, R.; Chamaillard, M.; Zouali, H.; Thomas, G.; Hugot, J.-P. CARD15 mutations in Blau syndrome. Nat. Genet. 2001, 29, 19-20. [CrossRef]

7. Inohara, N.; Nunez, G. NODs: Intracellular proteins involved in inflammation and apoptosis. Nat. Rev. Immunol. 2003, 3, 371-382. [CrossRef]

8. Hayden, M.S.; Ghosh, S. NF-кB in immunobiology. Cell Res. 2011, 21, 223-244. [CrossRef] [PubMed]

9. Hugot, J.-P.; Chamaillard, M.; Zouali, H.; Lesage, S.; Cézard, J.-P.; Belaiche, J.; Almer, S.; Tysk, C.; O’Morain, C.A.; Gassull, M.; et al. Association of NOD2 leucine-rich repeat variants with susceptibility to Crohn's disease. Nature 2001, 411, 599-603. [CrossRef]

10. Ogura, Y.; Bonen, D.K.; Inohara, N.; Nicolae, D.L.; Chen, F.F.; Ramos, R.; Britton, H.; Moran, T.; Karaliuskas, R.; Duerr, R.H.; et al. A frameshift mutation in NOD2 associated with susceptibility to Crohn's disease. Nature 2001, 411, 603-606. [CrossRef] [PubMed]

11. Chamaillard, M.; Philpott, D.; Girardin, S.E.; Zouali, H.; Lesage, S.; Chareyre, F.; Bui, T.H.; Giovannini, M.; Zaehringer, U.; Penard-Lacronique, V.; et al. Gene-environment interaction modulated by allelic heterogeneity in inflammatory deseases. Proc. Natl. Acad. Sci. USA 2003, 100, 3455-3460. [CrossRef]

12. Okafuji, I.; Nishikomori, R.; Kanazawa, N.; Kambe, N.; Fujisawa, A.; Yamazaki, S.; Saito, M.; Yoshioka, T.; Kawai, T.; Sakai, H.; et al. Role of the NOD2 genotype in the clinical phenotype of Blau syndrome and early-onset sarcoidosis. Arthritis Rheum. 2009, 60, 242-250. [CrossRef]

13. Yasui, K.; Yashiro, M.; Tsuge, M.; Manki, A.; Takemoto, K.; Yamamoto, M.; Morishima, T. Thalidomide dramatically improves the symptoms of early-onset sarcoidosis/Blau syndrome: Its possible action and mechanism. Arthritis Rheum. 2010, 62, $250-257$. [CrossRef]

14. Ackerman, A.B. Histologic Diagnosis of Inflammatory Skin Diseases.; Ardor Scribendi: New York, NY, USA, 2005; pp. 142-143.

15. Chen, E.S.; Moller, D.R. Etiologies of sarcoidosis. Clin. Rev. Allergy Immunol. 2015, 49, 6-18. [CrossRef]

16. Inaoka, P.; Shono, M.; Kamada, M.; Espinoza, J.L. Host-microbe interactions in the pathogenesis and clinical course of sarcoidosis. J. Miomed. Sci. 2019, 26, 45. [CrossRef]

17. Okamoto, H.; Mizuno, K.; Horio, T. Langhans-type and foreign-body-type multinucleated giant cells in cutaneous lesions of sarcoidosis. Acta Derm. Venereol. 2003, 83, 171-174. [CrossRef] [PubMed]

18. Fujisawa, A.; Kambe, N.; Saito, M.; Nishikomori, R.; Tanizaki, H.; Kanazawa, N.; Adachi, S.; Heike, T.; Sagara, J.; Suda, T.; et al. Disease-associated mutations in CIAS1 induce cathepsin B-dependent rapid cell death of human THP-1 monocytic cells. Blood 2007, 109, 2903-2911. [CrossRef]

19. Tsuchiya, S.; Kobayashi, Y.; Goto, Y.; Okumura, H.; Nakae, S.; Konno, T.; Tada, K. Induction of maturation in cultured human monocytic cells by a phorbol diester. Cancer Res. 1982, 42, 1530-1536. [PubMed]

20. Asseffa, A.; A Dickson, L.; Mohla, S.; A Bremner, T. Phorbol myristate acetate-differentiated THP-1 cells display increased levels of MHC class II mRNA and interferon- $\gamma$-inducible tumoricidal activity. Oncol. Res. 1993, 5, 11-18. [PubMed] 
21. Tsakadze, N.L.; Sithu, S.D.; Sen, U.; English, W.R.; Murphy, G.; D'Souza, S.E. Tumor necrosis factor- $\alpha$-converting enzyme (TACE/ADAM-17) mediates the ectodomain cleavage of intercellular adhesion molecule-1 (ICAM-1). J. Biol. Chem. 2006, 281, 3157-3164. [CrossRef] [PubMed]

22. Masumoto, J.; Yamazaki, T.; Ohta, K.; Nakayama, J.; Agematsu, K. Interleukin-1 $\beta$ suppression in Blau syndrome: Comment on the article by Martin et al. Arthritis Rheum. 2009, 60, 2544-2545. [CrossRef] [PubMed]

23. Kim, D.S.; Paik, S.H.; Lim, C.M.; Lee, S.D.; Koh, Y.; Kim, W.S.; Kim, W.D. Value of ICAM-1 expression and soluble ICAM-1 level as a marker of activity in sarcoidosis. Chest 1999, 115, 1059-1065. [CrossRef]

24. Van De Stolpe, A.; Caldenhoven, E.; Stade, B.G.; Koenderman, L.; A Raaijmakers, J.; Johnson, J.P.; Van Der Saag, P.T. 12-Otetradecanoylphorbol-13-acetate- and tumor necrosis factor alpha-mediated induction of intercellular adhesion molecule-1 is inhibited by dexamethasone. Functional analysis of the human intercellular adhesion molecular-1 promoter. J. Biol. Chem. 1994, 269, 6185-6192. [CrossRef]

25. Khachigian, L.M.; Resnick, N.; Gimbrone, M.A., Jr.; Collins, T. Nuclear factor-kappa B interacts functionally with the plateletderived growth factor B-chain shear-stress response element in vascular endothelial cells exposed to fluid shear stress. J. Clin. Investig. 1995, 96, 1169-1175. [CrossRef] [PubMed]

26. Marshall, B.G.; Wangoo, A.; Cook, H.T.; Shaw, R.J. Increased inflammatory cytokines and new collagen formation in cutaneous tuberculosis and sarcoidosis. Thorax 1996, 51, 1253-1261. [CrossRef] [PubMed]

27. Hoyle, G.W.; Li, J.; Finkelstein, J.B.; Eisenberg, T.; Liu, J.-Y.; Lasky, J.A.; Athas, G.; Morris, G.F.; Brody, A.R. Emphysematous lesions, inflammation, and fibrosis in the lungs of transgenic mice overexpressing platelet-derived growth factor. Am. J. Pathol. 1999, 154, 1763-1775. [CrossRef]

28. Janssen, C.E.; Rose, C.D.; De Hertogh, G.; Martin, T.M.; Meunier, B.B.; Cimaz, R.; Harjacek, M.; Quartier, P.; Cate, R.T.; Thomee, C.; et al. Morphologic and immunohistochemical characterization of granulomas in the nucleotide oligomerization domain 2-related disorders Blau syndrome and Crohn disease. J. Allergy Clin. Immunol. 2012, 129, 1076-1084. [CrossRef] [PubMed] 